\title{
Static shear modulus of electrorheological fluids
}

\author{
Lihong Shi, Wing Yim Tam,* Xianxiang Huang, and Ping Sheng \\ Department of Physics, The Hong Kong University of Science and Technology, Clear Water Bay, Kowloon, Hong Kong
}

(Received 26 July 2005; published 9 May 2006)

\begin{abstract}
We report measurements of the static shear modulus of electrorheological (ER) fluids consisting of waterwetted silica microspheres in silicone oil. A shear-annealing method, using creep-recovery $(\mathrm{CR})$ cycles under an external electric field, is used to enhance ER properties of the fluid. The shear-annealing method enables the silica spheres in the ER fluid to form better aligned and denser column microstructures. A stable state with elastic shear deformation is obtained after a sufficient number of CR cycles, with an optimal combination of stress duration and shear strength. Static shear modulus is obtained by measuring the elastic deformations at different shear stresses for an electric field frequency from 10 to $1000 \mathrm{~Hz}$. A water-bridge model is proposed to explain the enhanced shear modulus.
\end{abstract}

DOI: 10.1103/PhysRevE.73.051501

PACS number(s): 83.80.Gv, 62.10.+s, 83.60.La

\section{INTRODUCTION}

Electrorheological (ER) fluid is a complex fluid consisting of micron-sized dielectric particles dispersed in a nonconducting fluid. Under an external electric field the particles first form, in milliseconds, chains and then columns along the electric field direction. The formation of columns modifies the resistance of the fluid against shear stress, changing the fluid from a liquid to a "solid" [1]. The resistance against the shear depends on the strength of the electric field, the higher the field the stronger the resistance. However, once the electric field is removed the fluid will return to the liquid state, thus making the ER fluid an ideal material for real time feedback control applications in, e.g., isolation dampers, clutches, and valves [1]. The resistance to shears under an electric field manifests to a shear yield stress and modulus in elastic medium. While shear yield stress of ER fluids has been widely studied in both theories and experiments, the shear modulus has been limited mostly to theories [2-5]. It is only recently that the dynamical shear moduli $G^{\prime}$ and $G^{\prime \prime}$, are reported [6]. Measurements of static shear modulus have never been reported so far, to the best of our knowledge.

It is known that the microstructure in the columns formed from monodispersed spherical-particle ER fluids, as well as its counter part, the magnetorheological (MR) fluid, is a body-centered-tetragonal (BCT) lattice $[2,7,8]$. However, in real experiments, the formation of columns is far from the perfect BCT structure because they are formed from random particle aggregation, upon the application of an external electric field. This imperfection hinders the measurement of the static shear modulus. Figure 1(a) shows typical shear-strain measurements under constant shear stress for an ER system consisting of silica spheres in silicone oil with uniform initial mixing using a plate-plate configuration under an external electric field perpendicular to the plates. The shear stressstrain relation shows nonlinear behavior as shown in Fig. 1(b) with large scatter. The static shear modulus $G$ could be

\footnotetext{
*Corresponding author: Fax: 852-2358-1652, Email address: phtam@ust.hk.
}

inferred by a linear fit to the data at a small shear stress as indicated by the solid line in Fig. 1(b). However, the linear fit always shows nonzero intercept, in addition to large fluctuation between runs, indicating nonelastic behavior. This plasticity property can be demonstrated in a creep-recovery (CR) cycle as shown in Fig. 1(c) [9]. After the application of a stress the deformation cannot return to the origin in the recovery period indicating permanent deformation in the system. Furthermore, the deformation varies between runs. It is this permanent deformation that hinders the measurement of the static shear modulus.

Recently, we reported the use a shear-annealing method, using CR cycles, to enhance the strength of ER fluids [10]. The enhancement is due to the formation of better aligned and denser column microstructures after the application of $\mathrm{CR}$ cycles. Furthermore, it is possible to obtain stable states with elastic shear deformation after a sufficient number of CR cycles, with an optimal combination of stress duration and shear strength. Note that a similar enhancement due to strain hardening has also been observed in MR fluids [11]. Here we report, for the first time, the measurements of static shear modulus of ER fluids using the shear-annealing method to enhance the ER fluids with different water contents for electric field frequency from 10 to $1000 \mathrm{~Hz}$. We also obtain enhanced shear yield stress using the CR cycles. A simple water-bridge model is used to explain the enhanced shear ER effects. In what follows, details of the experiment are presented in Sec. II, followed by the water-bridge model to explain the static shear modulus in Sec. III. A discussion is presented in Sec. IV.

\section{EXPERIMENT}

The experiment was similar to that reported previously on the enhancement of ER yield stress by CR cycles [10]. $2.5 \pm 0.1$ micron water-wetted silica spheres were dispersed in silicone oil to form ER fluids for this experiment [12]. The silica spheres were first dried in an oven at $160^{\circ} \mathrm{C}$ for more than 12 days to completely remove any residual water. Then a known amount of water was added to the sample, by absorbing water moisture in open air before mixing with sili- 

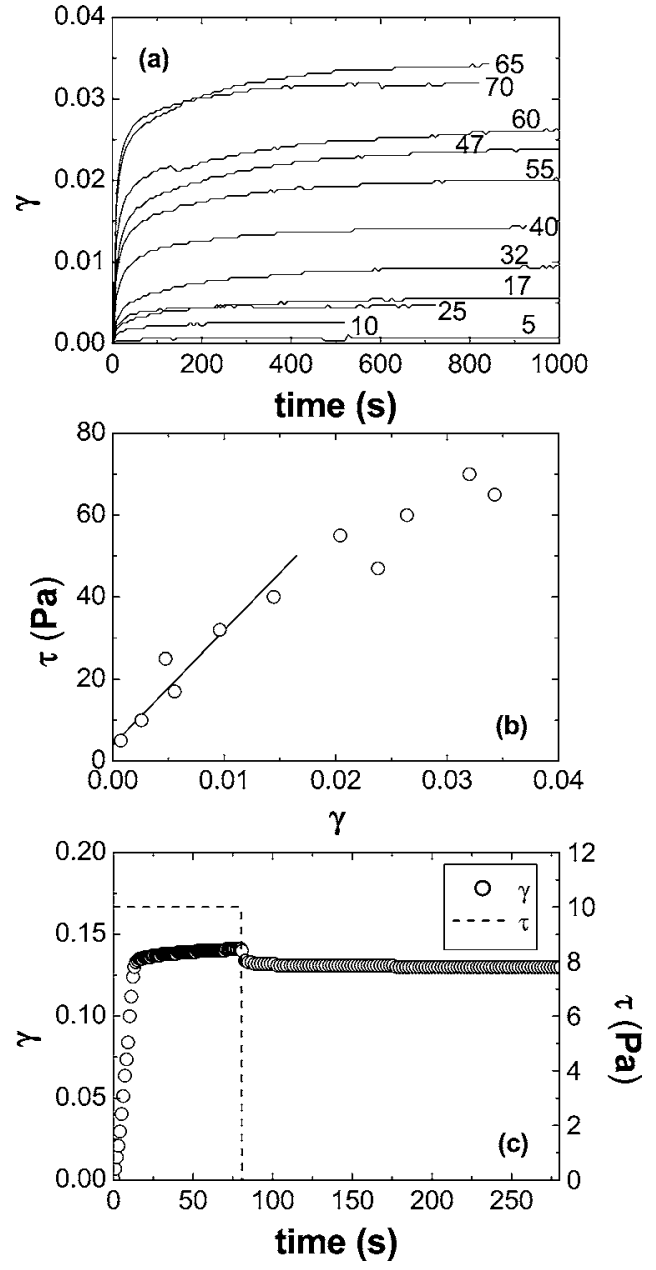

FIG. 1. (a) The strain $\gamma$ (deformation) of ER fluid containing a 0.35 volume fraction of 2.5 micron monodispersed silica spheres in silicon oil under a $1500 \mathrm{~Hz} 2.0 \mathrm{KV} / \mathrm{mm}$ electric field for different shear stresses indicated by the value for each curve. In the experiment, the ER fluid was remixed for each strain measurement under different stresses. (b) The corresponding shear stress-strain relation for (a). The solid line is a fit using data in the linear region. (c) A creep-recovery cycle showing the plastic deformation of the ER fluid.

cone oil, to increase the ER response. The final particle to oil volume fraction was 0.45 . A Hakke CS-20 rheometer with an ER option and parallel plate sensor was used for shear modulus and yield stress measurements. The ER fluid was first mixed using a shaker for half an hour to ensure uniform mixing. It was then placed between the parallel plates of the rheometer with the temperature regulated at $25^{\circ} \mathrm{C}$ by using a thermal circulator. The ac electric field, at $1.0 \mathrm{KV} / \mathrm{mm}$, was applied between the plates with $2 \mathrm{~mm}$ gap. The ER sample was "homogenized" at a constant strain rate for 1 min before applying the electric field. CR cycles in a control-stress mode were then applied to the sample after applying the electric field. One CR cycle consisted of a constant shear stress period and a recovery period without shear stress, keeping the applied electric field on. The effects of the strength of the shear stress, the shearing period, and the recovery period had been studied in a previous report [10].

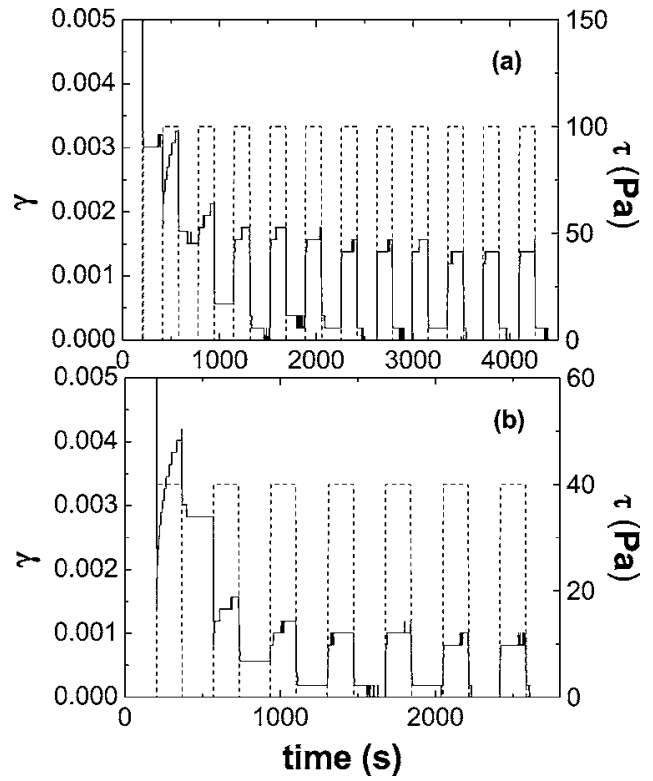

FIG. 2. CR cycles for ER fluid containing a 0.45 volume fraction of 2.5 micron silica spheres with (a) $6.5 \%$ and (b) $4.7 \%$ water in silicon oil under a $1000 \mathrm{~Hz} 1 \mathrm{KV} / \mathrm{mm}$ electric field using a shear and recovery periods of 200 and $300 \mathrm{~s}$. The shear stress used in the CR cycles is (a) $100 \mathrm{~Pa}$ and (b) $40 \mathrm{~Pa}$. Note that permanent deformations are resetted to zero before plotting the subsequence CR cycle for the beginning CR cycles. This is not needed for the ending CR cycles because the system became elastic after sufficient CR cycles.

Here we only varied the shear stress for different water contents and kept the shearing and recovery periods at $160 \mathrm{~s}$ and $200 \mathrm{~s}$, respectively. Figure 2 shows CR cycles with a $1000 \mathrm{~Hz} 1 \mathrm{KV} / \mathrm{mm}$ external electric field for static shear modulus (a) and shear yield stress (b) measurements. Note that in Fig. 2 the deformation was plotted by resetting it to zero after each CR cycle for cycles with permanent deformations. In the first few $\mathrm{CR}$ cycles large deformations were observed. However, it is clear that after a sufficient number of CR cycles, a steady state was obtained where the deformation returned to the origin in the recovery period in accord with previous results [10]. The number of CR cycles needed could vary slightly between runs and the annealing process was stopped only after the steady state was obtained. To obtain the static shear modulus, the deformation at the constant shear stress was measured in the low stress (linear) region after the $\mathrm{CR}$ cycles. For each strain measurement, the system was held at a shear stress for $300 \mathrm{~s}$ and then relaxed (removing the stress) to return to the origin. Another $300 \mathrm{~s}$ was waited before a second measurement began. Figure 3 shows the strains (elastic deformations) for different stresses at three frequencies, in descending order, for ER fluids with $6.5 \%$ water. All measurements were made at the linear region such that all deformations returned to the origin as clearly shown in Fig. 3. (The steps in the deformation represent the finite strain resolution of the rheometer.) At higher stresses, the deformation might not return to the origin in addition to the nonlinear behavior [see Fig. 1(b)] and thus were not attempted for static shear modulus measurements. Furthermore, runs were stopped once the system did not return to 


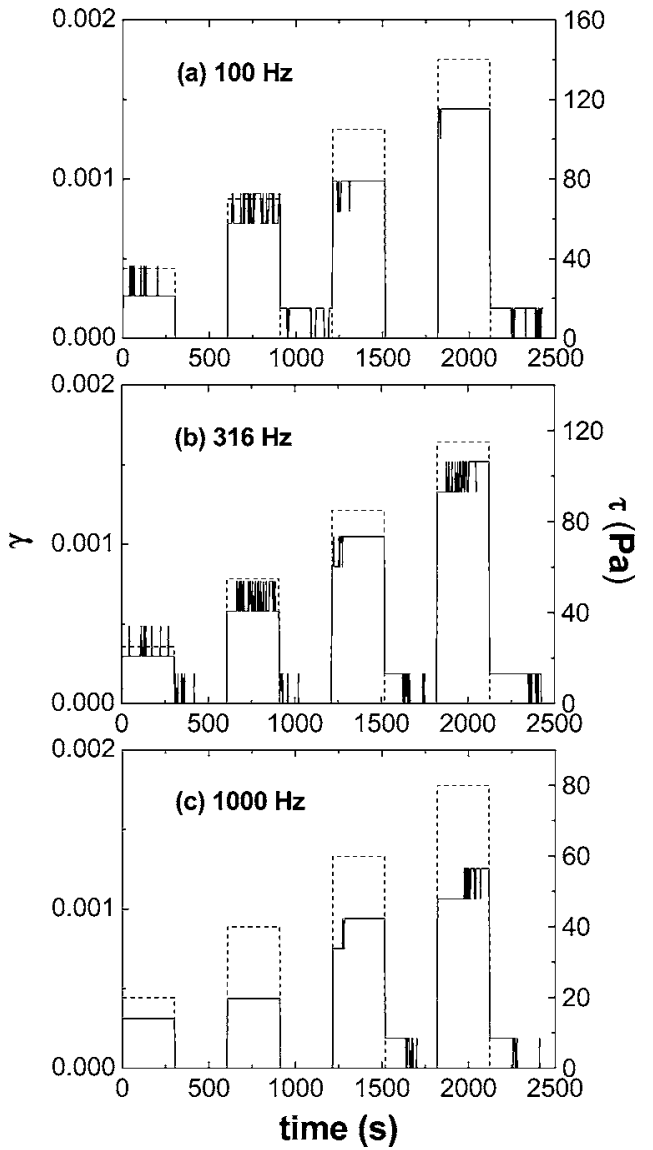

FIG. 3. The strain (elastic deformation) for CR cycles of different shear stress $\tau$ after the CR cycles in 2(a) for frequency (a) $100 \mathrm{~Hz}$, (b) $312 \mathrm{~Hz}$, and (c) $1000 \mathrm{~Hz}$. Note that there is no resetting of deformations.

the origin because it was important to keep the same state for a complete frequency sweep and any slipping (permanent deformation) in the columns would disrupt the original state, forbidding reliable measurements. Figure 4 shows the linear stress-strain relation at different frequencies for results in Fig. 3. Also shown are the linear fits (solid lines) to the data. The fits all pass the origin reflecting the elastic behavior of the system. Furthermore, the static shear modulus is simply given by the slope of the fits. Figure 5 shows the static shear

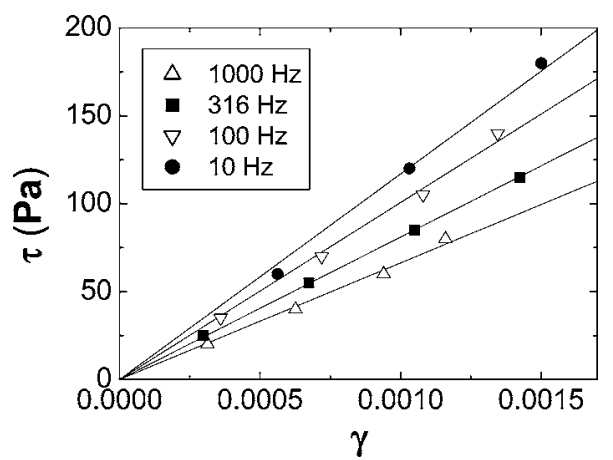

FIG. 4. The stress strain for the ER fluid in 2(a) for a different frequency. The solid lines are linear fits to the data whereby the static shear modulus is obtained.

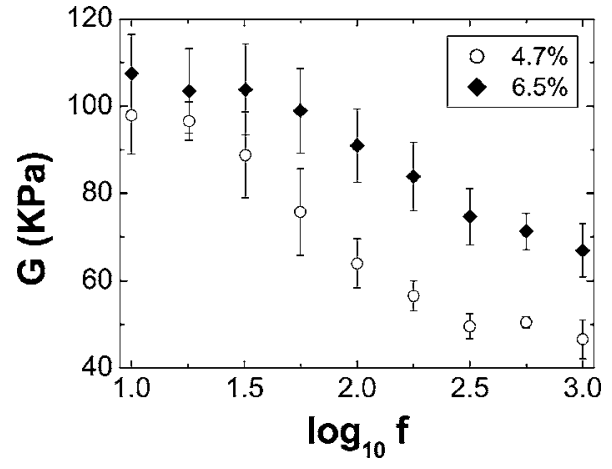

FIG. 5. The static shear modulus as a function of frequency for ER fluids with different water content.

modulus measured for two water contents of $4.7 \%$ and $6.5 \%$. The data are obtained by averaging several runs at the same conditions and the error bars are the standard deviations. Runs with a lower water content, e.g., below $4 \%$, showed too small deformations to be resolved by the rheometer with reasonable precision, resulting in large uncertainties in the shear modulus. This was the reason that a higher water content had to be used in order to obtain reliable results. The static shear modulus, increases with water content, shows a graduate decrease with frequency in agreement with previous calculations [5]. However, the magnitude of the modulus is one-two orders of magnitude larger than that predicted by the model calculation for similar systems [5].

In order to get a better comparison, the static yield stress was also measured. Like that in the static shear modulus, CR cycles were also applied as shown in Fig. 2(b) before the measurement. After the CR cycles, the ER fluid was sheared using a shear stress below the yield point and increased with a slow (in CS mode) ramping rate of $0.05 \mathrm{~Pa} / \mathrm{s}$ until the point when the fluid started to flow, as shown in Fig. 6 for three frequencies with $4.7 \%$ water. The shear yield stress was easily identified at the break point as indicated by the arrows in the figure. The ramping rate used was the largest rate determined with no ramping rate dependence. Once the fluid started to flow the original state would be destroyed. Thus the system had to be remixed and CR cycles were reapplied to prepare the initial state for a new measurement. Figure 7 shows the shear yield stress for ER fluids for 3.1, 4.7, and $6.5 \%$ water content. The results are again averaged over many runs and the error bars are the standard derivations of the averages. Again, the more the water content the higher the shear yield stress. In addition, the yield stress also decreases with a frequency with large enhancement, in agreement with previous measurements and theory $[3-5,10]$.

\section{MODEL}

It is known that water can enhance the performance of ER fluids from both experiments and theories because of the modification of the effective dielectric constant for a glassoil ER system [5,13]. There water plays only a passive role by modifying the effective dielectric constant. However, this effect alone is not enough to explain the dramatic enhancement in our results. In our case, because of the annealing 


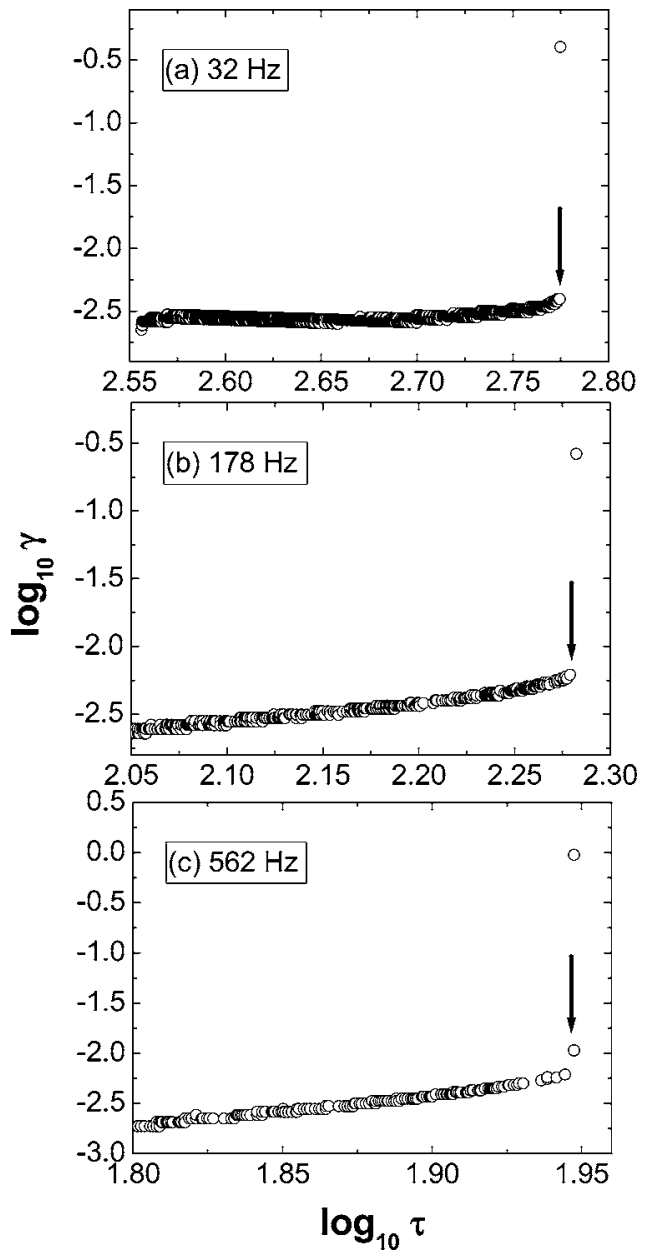

FIG. 6. Stress-strain relation of ER fluid containing a 0.45 volume fraction of 2.5 micron silica spheres with $4.7 \%$ water after CR cycles in 2(b) under (a) $32 \mathrm{~Hz}$, (b) $178 \mathrm{~Hz}$, and (c) $562 \mathrm{~Hz}$ $1 \mathrm{KV} / \mathrm{mm}$ electric field.

process, the water plays a more active role. In order to explain our experimental results, we propose the following physical picture. The small amount of water introduced into the sample can have such a large effect only if it coats the surfaces of the particles. Thus when two such coated particles are in close proximity the water coating can overlap and form a "water bridge" [14]. However, under random

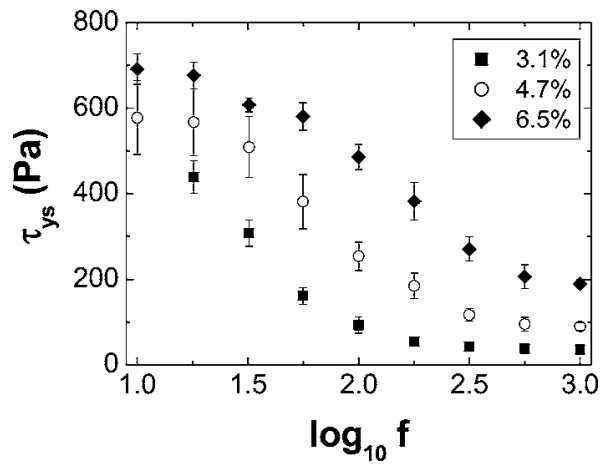

FIG. 7. Shear yield stress as a function of frequency for ER fluids with different water content.

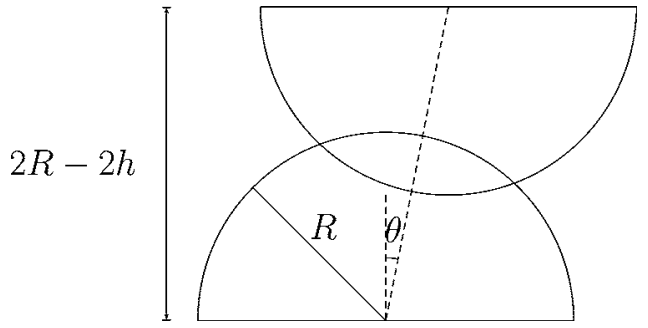

FIG. 8. Schematic diagram for the water-bridge model.

packing the particles together with their connecting water bridges can form at most a tenuous percolating network, able to sustain only a small shear stress. Only when the sample was subjected to a repeated cycling processing as described above in our shear-annealing method to achieve tight packing microstructures does it become likely that the waterbridge connections begin to form a homogeneous network. Under this picture, two elements would contribute to the shear modulus of the system: the oil-water interfacial tension (water-bridge mechanism) and the electrostatic polarization. Below we estimate the contribution of the interfacial component. It has to be noted that the shear modulus is measured by the stress associated with the distortion from the ground state microstructure. Hence a microstructure plays the role of (1) determining the density of solid particles, and (2) determining the allowed distortion(s). Here we assume a tight packing microstructure and the shear modulus is estimated from the basic distortion between two spheres.

Consider two semispheres (coated by a layer of water) tilted at an angle $\theta$ respect to the direction normal to shear as shown in Fig. 8. It is assumed that on average, there is one such water-bridge connection per sphere. The oil-water interfacial area for the two semispheres is given by

$$
S(\theta)=4 \pi R(R-h) / \cos (\theta),
$$

where $2 h$ is the overlap of two semispheres at $\theta=0$. The interfacial energy $W(\theta)=\sigma S(\theta)$, where $\sigma$ is the oil-water interfacial tension coefficient. At small shearing angle $\theta$,

$$
W(\theta)=4 \pi \sigma R(R-h)\left(1+\theta^{2} / 2\right) .
$$

By definition the shear modulus $G$ is given by

$$
\frac{1}{V} \frac{\partial W}{\partial \theta}=G \theta,
$$

in which $V$ is the volume and the left-hand-side is the stress. Hence

$$
G=4 \pi \sigma R(R-h) / V .
$$

For two contacting semispheres, $V \approx 4 \pi R^{3} / 3$, therefore

$$
G \approx \frac{3 \sigma}{R}\left(1-\frac{h}{R}\right)
$$

For oil-water interfaces, $\sigma \approx 30 \mathrm{mN} / \mathrm{m}$, and $R \gg h$ is taken as the radius of spheres $(1.25 \mu \mathrm{m})$. With these parameter values we obtain $G \approx 72 \mathrm{kPa}$, which is in excellent qualitative agreement with results. It should be noted that thermal motions of the solid particles can be neglected in the present 
case, as estimated by the ratio of electrical energy (per particle) to the thermal energy [15]:

$$
\lambda=\varepsilon_{f}(\beta E)^{2} r_{s}^{3} / 4 k_{B} T,
$$

where $r_{s}$ is the radius of particles, $\varepsilon_{f}$ is the dielectric constant of the fluid, and $T$ the temperature. Here $\beta$ denotes the polarizability of particles, i.e.,

$$
\beta=\left(\varepsilon_{s}-\varepsilon_{f}\right) /\left(\varepsilon_{s}+2 \varepsilon_{f}\right),
$$

$\varepsilon_{s}$ being the effective dielectric constant of solid particles. In the experiment, $r_{s}=1.25 \mu \mathrm{m}, \varepsilon_{f}=2$, and we assume $\varepsilon_{s}=2.5$. Under room temperature $T=300 \mathrm{~K}$ with an applied electric field $E=1000 \mathrm{~V} / \mathrm{mm}, \lambda=160$. In the above estimate, we have simply used $\varepsilon_{s}=2.5$ (that of glass) as the effective dielectric constant of the solid particles. Since water coating can only increase the particles' effective dielectric constant, the present estimate of $\lambda$ can be regarded as a lower bound. That is, the actual $\lambda$ value can be even larger than 160 .

\section{DISCUSSION}

The water-bridge model explains qualitatively the magnitude of the shear modulus in our experiment. It is noted that our measurements show a frequency-dependent shear modulus, varying by a factor of $\sim 2$ in Fig. 5. This frequency dependence can arise from the fact that there is indeed a dielectric constant sensitive component of the modulus, the theory of which has been detailed in a previous paper [16]. However, since this is not the main component responsible for the observed modulus, we show that a simple model based on a water-bridge mechanism can explain the experimental results quite well and yields a shear modulus with the same order $(72 \mathrm{kPa})$. Furthermore, the value obtained is likely to be a maximum because the effective water bridge per sphere could be less than 1 . More importantly, the waterbridge may not be able to form a regular compact network over the whole column structures in the ER fluid. Thus the actual shear modulus enhancement due to the water bridge is less than the predicted value. By combining with the electrostatic polarization contributions as calculated in pervious studies [3,5], the agreement between theory and experiment is far better than satisfactory. Furthermore, it would be desirable to use higher dielectric contrast particles in ER fluids, or use MR fluids, so that water could be eliminated to study the shear-annealing enhancement of shear modulus purely due to the rearrangement of microstructures.

To conclude we have measured, for the first time, the static shear modulus of ER fluids using a shear-annealing method to form well-aligned and denser column microstructures with elastic shear behavior. A water-bridge model is proposed to explain the enhanced shear modulus.

\section{ACKNOWLEDGMENTS}

This work was supported by the Competitive Earmarked Research Grant No. HKUST6122/98P of the Hong Kong Research Grant Council. Hong Kong Technical supports from K. C. Lau, H. W. Tsang, M. H. Kok, C. H. Wong, and W. T. Tse are acknowledged. We thank R. Ma for useful discussions.
[1] K. O. Havelka and F. E. Filisko, Progress in Electrorheology (Plenum Press, New York, 1995); W. M. Winslow, J. Appl. Phys. 20, 1137 (1949).

[2] G. Bossis, H. Clercx, Y. Grasselli, and E. Lemaice, in Electrorheological Fluids, edited by R. Tao and G. D. Roy (World Scientific, Singapore, 1994), p. 153; H. J. Clercx and G. Bossis, Phys. Rev. E 48, 2721 (1993); L. C. Davis, Phys. Rev. A 46, R719 (1992); H. Ma, W. Wen, W. Y. Tam, and P. Sheng, Phys. Rev. Lett. 77, 2499 (1996).

[3] W. Y. Tam, G. H. Yi, W. Wen, H. Ma, M. M. T. Loy, and P. Sheng, Phys. Rev. Lett. 78, 2987 (1997); C. W. Wu, Y. Chen, and H. Conrad, J. Phys. D 31, 960 (1998).

[4] L. C. Davis, J. Appl. Phys. 72, 1334 (1992).

[5] H. Ma, W. Wen, W. Y. Tam, and P. Sheng, Adv. Phys. 52, 343 (2003)

[6] S. G. Kim et al., J. Appl. Polym. Sci. 79, 108 (2001); B. D. Chin and H. H. Winter, Rheol. Acta 41, 265 (2002).

[7] R. Tao and J. M. Sun, Phys. Rev. Lett. 67, 398 (1991); Phys. Rev. A 44, R6181 (1991).

[8] W. Wen, N. Wang, H. Ma, Z. Lin, W. Y. Tam, C. T. Chan, and
P. Sheng, Phys. Rev. Lett. 82, 4248 (1999).

[9] The effect of the creep-recovery cycle on ER fluids has been reported recently, see, e.g., Y. Otsubo and K. Edamura, J. Colloid Interface Sci. 172, 530 (1995); , Colloids Surf., A 109, 63 (1996).

[10] K. C. Lau, L. Shi, W. Y. Tam, and P. Sheng, Phys. Rev. E 67, 052502 (2003).

[11] See, e.g., E. M. Furst and A. P. Gast, Phys. Rev. Lett. 82, 4130 (1999); X. Tang, X. Zhang, and R. Tao, J. Appl. Phys. 87, 2634 (2000).

[12] The 2.5 micron silica sphere was obtained from Nippon Shokubai and the silcone oil was \#705 from DOW Corning.

[13] W. Wen, H. Ma, W. Y. Tam, and P. Sheng, Phys. Rev. E 55, R1294 (1997).

[14] The water-bridge model was first proposed by F. E. Stangroom, Phys. Technol. 14, 290 (1983); see also H. See, H. Tamura, and M. Doi, J. Phys. D 26, 746 (1993).

[15] T. C. Halsey, Science 258, 761 (1992).

[16] H. Ma, W. Wen, W. Y. Tam, and P. Sheng, Phys. Rev. Lett. 77, 2499 (1996). 